\title{
ADHERENCE TO FACE PHOTOPROTECTION BEHAVIOURS IN XEREDERMA PIGMENTOSUM (XP) PATIENTS: INITIAL VALIDATION OF A NEW MEASURE
}

MARTHA CANFIELD ${ }^{1}$, SAM NORTON ${ }^{1}$, JESSICA WALBURN ${ }^{2}$, NATALIE MORRISONBOWEN ${ }^{1}$, KIRBY SAINSBURRY ${ }^{3}$, ROBERT SARKANY ${ }^{4}$, JOHN WEINMAN $^{2}$

\footnotetext{
${ }^{1}$ King's College London, Health Psychology Section, Psychology Department, Institute of Psychiatry, Psychology and Neuroscience, England, UK

${ }^{2}$ Institute of Pharmaceutical Science, King's College London, London, UK

${ }^{3}$ Institute of Health \& Society, Faculty of Medical Sciences, Newcastle University, Newcastle Upon Tyne, UK

${ }^{4}$ St John's Institute of Dermatology, Guy's and St. Thomas' NHS Foundation Trust, London, UK
}

Martha Canfield

Post-doc Research Associate

Department of Psychology

Institute of Psychiatry, Psychology and Neuroscience

King's College London

$5^{\text {th }}$ Floor Bermondsey Wing

Guy's Hospital

London SE1 9RT

Tel: +44 (0)20 71882606

Martha.canfield@kcl.ac.uk 


\begin{abstract}
Background: Few studies have examined photoprotection practices in individuals with rare genetic diseases, such as Xeroderma Pigmentosum (XP), where photoprotection is an important aspect of managing the condition. Currently no validated self-report tool exists for assessing adherence. Aims: We sought to validate a self-reported measure of adherence to face photoprotection behaviours in individuals diagnosed with XP. Methods: 66 XP patients recruited from the patient list of the XP specialist service in London, UK, completed a survey including self-reported adherence to specific photoprotection behaviours and wore a wrist-worn UVR electronic dosimeter for 21 days while also completing a daily UVR photoprotection diary. Internal reliability and convergent validity of the measure was explored in relation to self-reported UVR photoprotection practices, clinical variables, and objective measures of UVR protection. Results: Internal consistency of the self-reported scale was satisfactory. Correlations between the adherence to photoprotection measure with other photoprotection practices and clinical variables ranged from small to large in size. There was general agreement between the self-reported measure and diary and dosimeter measures as the mean scores for levels of adherence to photoprotection increased with average day protection. However, there were outliers specifically at the higher ends of average day protection and poor agreement between levels of adherence to the face photoprotection and the amount of UVR dose reaching the face. Conclusions: Findings showed that our measure may provide a standardised and internally reliable self-reported assessment of UVR protection. However, given the potential low sensitivity to detect high adherence, we encourage future studies to combine objective and subjective reports to increase the confidence in the accuracy of the measure of adherence to face photoprotection.
\end{abstract}

Key words: Xeroderma Pigmentosum, photoprotection, self-reported measure, validation 


\section{INTRODUCTION}

Xeroderma Pigmentosum $(\mathrm{XP})$ is rare autosomal recessive genetic disorder affecting 1 in 250,000 people that results in deficiencies in the genetic pathways responsible for repair of DNA damage caused by exposure to ultraviolet radiation (UVR). The main clinical manifestations of XP include skin damage, increased risk of skin cancer, ocular damage and neurological deterioration. Skin cancer is the major cause of premature death in people diagnosed with XP, with the median age of death around 32 years old (Bradford, 2010).

While there is no cure for $\mathrm{XP}$, the adoption of rigorous photoprotection behaviours can reduce the likelihood of poor skin and ocular problems developing and thus improve prognosis. The clinical guidance is for people with XP try to minimise their exposure to UVR. This can be achieved by avoiding spending time outside, particularly during the middle of the day when UVR levels are highest, and while outside applying sunscreen and lip block, plus wearing photoprotective clothing (e.g. ideally wearing a UVR protective face visor, trousers, long sleeved top, and gloves). Potentially due to the rare nature of XP, few studies have explored the use of photoprotection in this population, and little is known about rates of photoprotection adherence or the key drivers of adherence to photoprotection (Walburn et al., 2017).

Research into photoprotection is predominantly focused on healthy populations and individuals with, or at increased risk of, skin cancer (Geller et al, 2002; Dennis et al., 2003; Ghiasvand et al., 2016; Green et al., 2013). Photoprotection is complex and thus difficult to measure in a simple way since it relies on several separate behaviours. Often studies use objective measures of UVR exposure (electronic or polysulfone film badge dosimetry), observations of protective behaviours, such as the application of sunscreen, or self-report diaries of protective behaviours (Andersen et al. 2010; Dobbinson et al., 2009; Stanton et al. 2003; O'Riordan et al. 2006). Although providing useful information, their intrusiveness and the burden on respondents to adhere to monitoring, which may itself influence the 
adoption of photoprotection behaviours, may make these techniques unreliable and impractical outside of research studies (Dobbinson et al., 2014).

Several self-report surveys assessing specific aspects of photoprotection have been used. These include asking participants for estimates of total sun exposure on an average weekday and weekend day (Diffey\& Norridge, 2009), asking about consistency of use of sunscreen, sunglasses, and clothing (Hillhouse et al., 2012; James et al., 2002), frequency of sunbathing (Mahler, 2008; Pettijohn, 2009) and how easily they burn (Dennis et al., 2008; Hill, Dobbinson \& Makin, 2009). There have only been limited attempts to validate self-report measures of photoprotection against objective measures of UVR exposure, but these have been largely promising in their findings (Dobbinson et al., 2014; Glanz \& Mayer 2005; Hillhouse et al., 2012; O'riordan et al., 2009). A key issue for the use of these tools in populations with generally low UVR exposure, such as XP, is that they are unlikely to be sensitive to the detection of small but important differences in photoprotection practices, particularly at specific clinically important areas such as the face (Morel \& Cano, 2017; Sainsbury et al., 2017). The only existing tool for use in this population is clinician-reported and has not been fully validated (Henshaw \& Turner, 2016). As such, there is a need to develop and validate a new self-report survey for people with XP.

The aim of this study was to evaluate the validity of a new self-report survey of adherence to photoprotection behaviours relevant to protecting the face in individuals diagnosed with XP. The focus on the face is due to the predominant occurrence of skin cancers in XP populations in this area (Kraemer et al., 1994). Convergent validity was explored in relation to electronic UVR dosimetry, a daily photoprotection diary, and clinicians rating of photoprotection. 


\section{METHODS}

\section{Participants and procedure}

Participants were 66 patients diagnosed with XP, recruited from the National XP specialist service at Guy's \& St Thomas' NHS Foundation Trust. All participants were recruited as part of a larger mixed-methods study of photoprotection in people with XP (Walburn et al., 2017). Individuals of any age were eligible to participate if they had a confirmed diagnosis of XP. Thirty-nine (59\%) participants were adults or older adolescents (aged 16 or above) who were responsible for their own photoprotection behaviour. The remaining $27(41 \%)$ participants consisted of children, younger adolescents, and cognitively impaired individuals where an adult was partly or entirely responsible for photoprotection for the individual. Although these individuals wore the UVR dosimeter, the daily photoprotection diary and questionnaire were completed by the person responsible for photoprotection -- typically a parent. This group are henceforth referred to as carer-report sample.

The research nurse approached eligible individuals attending outpatient clinic appointments in a three-month period starting in late 2016. Prior to taking informed consent the research nurse verbally explained the study to eligible participants and provided a study information sheet. For those patients who were not due to attend clinic within the recruitment period, an information pack containing a cover letter and study information sheet, was sent to their home address. Approval to conduct this study was granted by Camden and King's Cross Research Ethics Committee 15/LO/1395.

\section{Measures}

Adherence to face photoprotection: With input from the XP clinical team, a scale was developed comprising of fourteen-items asking how often in the past seven-days participants wore or used each of the following seven forms of facial photoprotection: UVR protective visor, brimmed-hat, glasses with UVR protection, sunscreen applied to the face, lip sunblock, scarf or face buff covering the face, and a hooded jumper with the hood worn up. Each item 
was asked in reference to both cloudy and sunny days, as behaviour is likely to differ under these conditions. Each item was scored on a five-point ordinal scale from 1 (Never) to 5 (Always).

A sum-score across behaviours is not appropriate since there is potentially a negative association between behaviours. That is, using one form of protection (e.g., face visor) makes doing another less likely (e.g., wearing a scarf underneath the visor is unnecessary). Therefore, an algorithm for scoring adherence to photoprotection behaviours was generated based on the likely effectiveness of the photoprotection afforded by the different behaviours (see supplementary material). Specifically, the seven individual behaviours were combined to form photoprotection scores based on five regions of the face (Figure 1): i) forehead, which is protected by the visor, hat, hoodie, and sunscreen; ii) lower face, which is protected by the visor, scarf, sunscreen, and lip sunblock; iii) nose, which is protected by the visor and sunscreen; iv) cheeks and side of face, which is protected by the visor and sunscreen; and v) eyes, which is protected by the visor and glasses.

Protection scores for each region are calculated based on the response to wearing a UVR protective visor, and then adjusted based on the responses to other behaviours relating to that region where the score for the visor was less than 5 (always). Full details of the scoring algorithm are provided in the supplementary material. The logic for this is that wearing a UVR protective visor protects against virtually the entire solar UVA/UVB range, and is considered optimal clinically, therefore, where a visor is indicated to have always been worn, the score assigned is the maximum 5 for each region. Where a visor is indicated as being worn less often, the score for the visor can be adjusted upwards based on responses to other photoprotective behaviours. For example, where a visor is reported as never being worn but the respondent indicates they always wore a hat and/or hoodie (forehead), a scarf (lower face) and glasses (eyes) a score of five is given for each of these regions since close to $100 \%$ of UVR will be protected by these items. Since sunscreen and lip sunblock protects against around $70 \%$ of UVR (based on SPF50 worn at $50 \%$ recommended thickness; 
Young, 2017), thus where the score for the region is less than 5 based on other behaviours, the maximum score is restricted to 4 even where sunscreen and lip sunblock are indicated as always being used.

The scores for each region are summed separately for cloudy and sunny days to give a scores for the entire face for each scenario ranging between 5 and 25, where higher scores indicate better photoprotection. The scores for cloudy and sunny days are averaged to give an overall photoprotection score. Although rather arbitrary, we suggested the following labels for describing different scores: 5 indicates no use of photoprotection; 6 to 10 indicates very low use of photoprotection; 11 to 15 indicates low use of photoprotection; 16 to 20 indicates moderate use of photoprotection; 21 to 24 indicates good use of photoprotection; and 25 indicates 5 excellent use of photoprotection. In the case of XP any score lower than 25 is considered suboptimal, with scores of 20 or lower being considered inadequate.

Adherence to body photoprotection: A ten-item scale asking how often in the last sevendays participants used five photoprotective behaviours for other parts of the body on cloudy and sunny days was also developed. Each item was scored on a five-point ordinal scale from 1 (Never) to 5 (Always). Adherence to body photoprotection behaviours was defined by the sum of scores across two body's areas: arms and upper torso (long sleeved top, gloves and sunscreen applied to the arms and hands) and legs (long trousers/skirt and sunscreen applied to the legs). The score for each region was based on the response for wearing a long-sleeved top or long trousers/skirt and increased by the response to sunscreen use. As with the face, where the score for a region is less than 5 based on other behaviours, the maximum score is restricted to 4 even where sunscreen and lip sunblock are indicated as always being used. Scores for the two body areas for both cloudy and sunny days are summed and divided by two to create a total score. The mean across the sum-scores for cloudy and sunny days can be used as an overall body photoprotection score. 
Internal reliability for the body scale was high for cloudy and sunny days .88 and .84 , respectively.

Avoidance of going outside and frequency of photoprotection whilst outside: Participants were asked single items to report how often they avoided going outside and how they had protected themselves against UVR when they went outside in the last seven days.

Responses were given separately for cloudy and sunny days on a five-point scale, ranging from 1 (never) to 5 (always).

Daily UVR protection diary: A daily UVR protection diary was used to collect information on time spent outside, specific photoprotection behaviours, and the type of outdoor activity that participants were involved in. It is based on an adapted version of the UK Office of National Statistics Time Use Survey (Gershuny, 2011) and was completed daily for 50 days. For this study, only the 21 days matching the period where the UVR dosimeter was worn were used. Detailed information about the diary can be found elsewhere (Walburn et al., 2017).

Objective UVR measure: The amount of UVR exposure was measured by a wristworn UVR electronic dosimeter (SunSaver 3, Bispebjerg Hospital, Copenhagen, Denmark). The dosimeters provide real time measurements of the UV level in the patients' environment by measuring UVR exposure (standard erythemal dose, SED), movement (number of movements) and temperature $\left({ }^{\circ} \mathrm{C}\right)$ every 5 seconds. Due to the memory capacity, the dosimeter was set to record the mean for each of these variables across intervals set at 5 minutes. Participants wore the dosimeter for the first 21 days of completing the daily UVR protection diary. For the purpose of this analysis, two outcome variables were tested: (1) the average day UVR exposure, and (2) average day UVR dose to the face per day after adjusting for photoprotection practices reported in the daily UVR protection diary. Further information about the dosimeter and the calculation of the adjusted dose to the face can be found in Walburn et al. (2017). 
Demographic and clinical variables: Self-reported information on participants' age, gender, educational level and skin colour were collected. Participants were additionally asked to report age at the time of diagnosis, whether they ever had a skin cancer, experience of cognitive impairments (hearing, walking or speaking) caused by $\mathrm{XP}$, and/or eyes problems, and how easily they sunburn using a Likert Scale ranging from 0 (not easily) to 3 (extremely easy). A binary burning variable was created based how easily their sunburn ( 0 or 1 not easily and 2 or 3 easily). A clinical rating of overall UVR protection was also obtained from patient's notes at the XP clinic database. This rating, on a 1 to 20 scale, where higher scores indicate photoprotection is made by a specialist nurse based on their judgment of a patientsenvironmental, physical, and chemical UVR protection.

\section{Statistical analyses}

Internal-consistency of the sum-score of the five face region variables for cloudy and sunny days was assessed by examining the correlation between the score for each region and the total score, plus Cronbach's alpha as an estimate of relaibility. Responses for cloudy and sunny days were considered separately. Convergent validity between the sum-scores for face photoprotection on cloudy and sunny days was assessed by examining correlations with other indicators of photoprotection: mean daily UVR exposure (dosimetry), proportion of UVR protected against during the day (photoprotection diary), mean daily UVR dose to the face (combination of dosimetry and diary), and the self-reports for the frequency of avoidance of going outside and photoprotection whilst outside in the last 7 days. Pearson correlations were estimated where both variables were continuous and polyserial correlation estimated where one of the items was ordinal. Recruiting a sufficiently sized sample for analyses to have acceptable power is prohibitive given the rarity of $\mathrm{XP}$, thus we focus we focus interpretation on the magnitude of the correlations rather than significance tests. Specifically we use the following rules of thumb: $\geq .10$ for a small effect, $\geq .30$ for a medium effect, and $\geq .50$ for a large effect (Cohen, 1988). 


\section{RESULTS}

Table 1 presents the sample characteristics, which were typical of the wider XP population.

Table 2 shows means and standard deviations for each of the face photoprotection behaviours on cloudy and sunny days. Not unsurprisingly, most behaviours were undertaken more frequently on sunny rather than cloudy days. The rank ordering of behaviours remained stable across both cloudy and sunny days with the most frequently used photoprotective behaviour being the application of sunscreen, followed by wearing glasses and a hat.

Internal consistency was satisfactory for adherence to photoprotection for cloudy days $(\alpha=$ $.87)$, for sunny days $(\alpha=.81)$ and for the average adherence on cloudy and sunny days ( $\alpha=$ .82). Inter-item and item-test correlations between the five areas were all greater than 0.50 .

Figure 2 shows the proportion within each of the photoprotection categories for cloudy and sunny days. The sum-scores were left skewed with approximately one-third scoring at the maximum on both cloudy and sunny days. The scale was found to be able to reliably identify differences in photoprotection between cloudy and sunny days and between those where the responses were self-reported or reported by a carer (Figure 3). Specifically, a mixedeffects model indicated a significant main effect for self- versus carer-report $(z=6.2, p<.001)$, a significant main-effect for sunny versus cloudy days $(z=5.1, p<.001$, and a significant interaction $(z=2.33, p=.020)$.

Face photoprotection was found to have good convergent validity in relation to other measures of photoprotection (Table 3). Very strong positive correlations were observed between face photoprotection on cloudy and sunny days with both self-reported frequency of photoprotection while outside in the past seven days and the clinician rating of overall photoprotection. Strong positive correlations were observed between face photoprotection and body photoprotection. Correlations between face photoprotection and the mean proportion of face photoprotection estimated from the daily photoprotection diary were also 
strong positive. However, there was no correlation observed between the level of face photoprotection with self-reported avoidance of going outside in the past seven days, and while the association with the mean daily facial UVR exposure (combining the photoprotection diary and dosimetry data) was negative as expected, the effect was relatively weak.

Levels of agreement between adherence to face photoprotection categories on cloudy and sunny days with the daily photoprotection diary and dosimetry are shown in Figure 4. The overall pattern is for mean daily face photoprotection to increase, and to some extent facial UVR exposure to decrease, with increasing categories of face photoprotection. However, while participants reporting low or very low face photoprotection were unlikely to have high levels of average daily protection, not all participants who reported having good or excellent face photoprotection were observed to have high levels of photoprotection in the daily diary. This suggests the tool may be sensitive to detect low adherence to face photoprotection but not high adherence.

\section{DISCUSSION}

This study reports the initial evaluation of a self-report tool for assessing face photoprotection in individuals diagnosed with XP. This simple self-report measure was shown to be reliable and have good convergent validity with other indicators of photoprotection. In particular, it corresponded well with a daily photoprotection diary and, therefore, may be useful in situations where a daily diary is impractical, particularly where the aim is to identify people with low adherence to photoprotection recommendations. However, given the potential low sensitivity to detect high levels of photoprotection and the low correlation with facial UVR exposure, we encourage future researchers to combine dosimetry with subjective reports of photoprotection practice where possible. 
Few studies have reported comparisons between self-report measures of photoprotection and objective and clinical measures (Glanz \&Mayer, 2005; Dobbinson et al., 2014; O'Riordan et al., 2009). This study further demonstrates that self-report measures can have can be used to assess UVR protective practices. Furthermore, given this tool is designed to assess full UVR coverage of the face, it provides a promising self-reported outcome measure for the photodermatological field. To date, it remains unclear how effective self-reported measures are in detecting whether the behaviour adopted does fully protect the target region. For example, participants are often asked to report the frequency of hat use without exploring whether they are concurrently protecting other regions of the face, or the type of hat used, which will impact the coverage achieved (e.g., a cap vs. a wide-brimmed hat). Reviews of the literature have recognised that UVR protection can occur in different forms and its efficacy usually depends on the adoption of more than one photoprotection behaviour (Green et al., 2013; Diaz \& Nesbitt, 2013). Evidence also suggests that clothing and seeking shade are more effective than sunscreen in protecting against UVR (Autier Boniol \& Dore, 2007; Linos et al., 2011; Thieden et al., 2005). Measures that account for the extent to which regions of the body are being simultaneously protected, and therefore, by extrapolation, the level of importance of the behaviours adopted, are important as this may provide a better understanding of adherence to photoprotection behaviours.

A limitation with the face photoprotection measure is the ceiling effect, with over onethird of respondents achieving the highest score - mainly due to reporting having 'always' worn a UVR protective visor while outside. Comparison with the photoprotection diary indicated that for some people self-reporting excellent face photoprotection actual photoprotection was likely to be as low as $50 \%$. This difference may be due to socially desirable responding, or alternatively may reflect differences in interpretation of the term 'always'. For example, it is possible for someone to wear a face visor on every occasion they go outside but not necessarily wear it for the entire duration of the time outside on every occasion. 
A further limitation is the relatively small size of the sample. However, XP is a rare disease and the sample recruited for this study represents over half of the known cases in the UK. Recruitment of sample sizes required for full psychometric assessment with typical levels of precision are simply not feasible. Although a larger sample size is not possible, we did assess validity against a range of other tools, which provides confidence that the measure is valid and reliable.

\section{Conclusion}

The purpose of this study was to test the validity and reliability of a measure of adherence to face photoprotection in XP population. Findings shown that our measure may provide a standardised and internally reliable self-reported assessment of UVR protection, which may be particularly useful in assessing intervention outcomes aimed at improving photoprotection practices. However, we encourage the combined use of objective measures within our selfreported tool to increase the confidence in the accuracy of the outcome measure of adherence to face photoprotection. The adherence of face photoprotection behaviour measure would benefit from future studies exploring further construct validity.

\section{Funding}

This article presents independent research funded by the National Institute for Health Research (NIHR) under its Programme Grants for Applied Research scheme (RP-PG-121220009). The views expressed are those of the authors and not necessarily those of the NHS, the NIHR or the Department of Health. 


\section{REFERENCES}

Andersen, P.A., Buller, D.B., Walkosz, B.J., Scott, M.D., Maloy, J.A., Cutter, G.R., \& Dignan, M.D. (2010). Environmental cues to UV radiation and personal sun protection in outdoor winter recreation. Archives of Dermatology, 146,1241-1247.

Autier, P., Boniol, M., \& Dore, J. (2007). Sunscreen use and increased duration of intentional sun exposure: Still a burning issue. International Journal of Cancer, 121.

Cohen, J. (1988). Statistical power analysis for the behavioral sciences. $2^{\text {nd }}$ Edition. Hillsdale, NJ: Lawrence Erlbaum Associators.

Dennis L.K., Freeman L.E.B, VanBeek, M.J. (2003). Sunscreen use and the risk for melanoma: a quantitative review. Annal of Internal Medicine, 139, 966 -978.

Dennis, L.K., Vanbeek, M. J., Freeman, L.E.B., Smith, B. J., Dawson, D.V., \& Coughlin, A. C. (2008). Sunburns and risk of cutaneous melanoma: does age matter? A comprehensive meta-analysis. Annals of Epidemiology, 18, 614-627.

Diaz, J. H., \& Nesbitt, Jr. (2013). Sun Exposure Behavior and Protection: Recommendations for Travelers. Journal of Travel Medicine, 20, 108-118.

Diffey, B.L., \& Norridge, Z. (2009). Reported sun exposure, attitudes to sun protection and perceptions of skin cancer risk: a survey of visitors to Cancer Research UK's SunSmart campaign website. British Journal of Dermatology, 160, 1292-1298.

Dobbinson, S.J., Jamsen, K., Dixon, H.G., Spittal, M.J., Lagerlund, M., Lipscomb, J.E., ... \& Hill, D. J. (2014). Assessing population-wide behaviour change: concordance of 10year trends in self-reported and observed sun protection. International Journal of Public Health, 59, 157-166.

Dobbinson, S.J., White, V., Wakefield, M.A., Jamsen, K.M., White, V., Livingston, P.M., English, D.R., \& Simpson, J. A. (2009) Adolescents' use of purpose built shade in secondary schools: cluster randomised controlled trial. BMJ 338:b95.

Geller, A.C., Colditz, G., Oliveria, S., Emmons K., Jorgensen C., Aweh, G. N., \& Frazier, L. (2002). Use of Sunscreen, Sunburning Rates, and Tanning Bed Use Among More Than 10000 US Children and Adolescents. Pediatrics, 109, 1009-1014.

Gershuny, J. (2011). Time-use surveys and the measurement of national well-being. Oxford, UK: Centre for Time Use Research, University of Oxford.

Glanz, K., \& Mayer, J.A. (2005). Reducing ultraviolet radiation exposure to prevent skin cancer methodology and measurement. American Journal of Preventive Medicine, 29, 131-142.

Ghiasvand R., Weiderpass E., Green A.C., Lund E., \& Veirerod M. B. Sunscreen use and subsequent melanoma risk: A population-based cohor study. Journal of Clinical Oncology, 34, 1-8.

Green, A.C., Willians, G.M., Logan V., \& Strutton, G. (2010). Reduced Melanoma After Regular Sunscreen Use: Randomized Trial Follow-Up. Journal of Clinical Oncology, 29, 257-263.

Green, A.C., Williams G.M., \& Point. (2007). Sunscreen use is a safe and effective approach to skin cancer prevention. Cancer Epidemiol Biomarkers Prevention, 16, 1921-1922. 
Henshaw T, Turner S (2016). Audit of ultraviolet radiation practices by patients with xeroderma pigmentosum. Dermatological Nursing 15 (3): 65

Hill, D.J, Dobbinson, S.J., Makin J.K. (2009). Interventions to lower ultraviolet radiation exposure: Education, legislation, and public policy. In: 2009 Educational Book Category: Melanoma/Skin Cancers. Alexandria: American Society of Clinical Oncology.

Hillhouse, J., Turrisi, R., Jaccard, J., \& Robinson, J. (2012). Accuracy of Self-Reported Sun Exposure and Sun Protection Behavior. Prevention Science, 13, 519-531.

James, A.S., Tripp, M.K., Parcel, G.S., Sweeney, A., \& Gritz, E.R. (2002). Psychosocial correlates of sun-protective practices of preschool staff toward their students. Health Education Research, 17, 305-314.

Linos, E., Keiser, E., Fu, T., Colditz G., Chen S., \& Tang J. Y. (2011). Hat, shade, long sleeves, or sunscreen? Rethinking US sun protection messages based on their relative effectiveness. Cancer, Causes \& Control, 22, 1067-1071.

Kraemer, K. H., Lee, M. M., Andrews, A. D., \& Lambert, W. C. (1994). The role of sunlight and DNA repair in melanoma and nonmelanoma skin cacner. The xeroderma pigmentosum paradigm. Achives of Dermatology, 130(8), 1018-1021. doi: 10.1001/archderm.1994.01690080084012

Mahler, H.I., Kulik, J.A., Butler, H.A., Gerrard, M., \& Gibbons, F.X. (2008). Social norms information enhances the efficacy of an appearance-based sun protection intervention. Social Science \& Medicine, 67, 321-329.

Morel, T., \& Cano, S. J. (2017). Measuring what matters to rare disease patients reflections on the work by the IRDiRC taskforce on patient-centered outcome measures. Orphanet Journal of Rare Dieases, 12, 171.

Pettijohn, I.I., Pettijohn, T.F., \& Geschke K. S. (2009). Changes in Sun Tanning Attitudes and Behaviors of US College Students from 1995 to 2005. College Student Journal, 43, 161-165.

O'Riordan, D.L., Nehl, E., Gies, P., Bundy, L., Burgess, K., Davis, E., \& Glanz, K. (2009). Validity of covering-up sun-protection habits: Association of observations and selfreport. Journal of the American Academy of Dermatology, 60, 739-744.

Sainsbury, K., Walburn, J., Araujo-Soares, V. \& Weinman, J. (2017). Challenges and proposed solutions for formative research to inform systematic intervention development in rare and unstudied conditions: The case example of Xeroderma Pigmentosum. British Journal of Health Psychology. Editorial.

Stanton, W.R., Saleheen, H.N., O'Riordan, D., Roy, C.R. (2003). Environmental conditions and variation in levels of sun exposure among children in child care. International Journal of Behavioral Medicine, 10, 285-229.

Thieden, E., Philipsen, P.A., Sandby-Moller, J., \& Wulf, H.C. (2005). Sunburn Related to UV Radiation Exposure, Age, Sex, Occupation, and Sun Bed Use Based on TimeStamped Personal Dosimetry and Sun Behavior Diaries. Archives of Dermatology, 141,482-488. 
Walburn, J., Sarkany, R., Norton, S., Foster, L., Morgan, M., Sainsbury, K., ... \& Sniehotta, F. F. . (2017). An investigation of the predictors of photoprotection and UVR dose to the face in patients with XP: a protocol using observational mixed methods. BMJ Open, 7, e018364.

Young, A.R., Claveau, J., \& Rossi, A. B. (2017). Ultraviolet radiation and the skin:

Photobiology and sunscreen photoprotection. Journal of the American Academy of Dermatology, 76, S100-S109. 
Table 1. Sample characteristics $(N=66)$

\begin{tabular}{lr}
\hline Characteristics & \\
\hline Male, $n(\%)$ & $36(54.55 \%)$ \\
Age, mean (S.D.) & $50(73.6 \%)$ \\
White skin colour, $n(\%)$ & $19(28.78)$ \\
Too young for having school qualifications, $n(\%)$ & $27(40.01)$ \\
Reported by the carer, $n(\%)$ & $36(54.55)$ \\
Worn dosimeter, $n(\%)$ & $15.15(2.11)$ \\
Age at the time of diagnose, mean (S.D.) & $32(48.48 \%)$ \\
Burner $n(\%)$ & $28(42.42 \%)$ \\
Skin Cancer $n(\%)$ & $17(25.76 \%)$ \\
XP causing cognitive problems, $n(\%)$ & $43(65.15 \%)$ \\
Eyes problems, $n(\%)$ &
\end{tabular}


Table 2. Means (standard deviations) for the frequency of each face photoprotection behaviour $(\mathrm{N}=66)$

\begin{tabular}{lcc}
\hline $\begin{array}{l}\text { How often you do the different things } \\
\text { on the days when it is cloudy and } \\
\text { then on the days when it is sunny, }\end{array}$ & Cloudy days & Sunny days \\
throughout the year? & & \\
\hline Do you wear a UVR protective visor? & $2.48(1.85)$ & $2.62(1.92)$ \\
Do you wear a hat? & $3.55(1.60)$ & $4.06(1.39)$ \\
Do you wear glasses? & $3.79(1.47)$ & $4.29(1.26)$ \\
Do you wear a scarf or "face buff"? & $2.17(1.51)$ & $2.17(1.53)$ \\
Do you wear a hoodie (worn-up)? & $2.28(1.49)$ & $2.35(1.53)$ \\
Do you use sunscreen on your face? & $4.26(1.14)$ & $4.61(0.82)$ \\
Do you put on lip sunblock? & $2.85(1.66)$ & $3.15(1.64)$ \\
\hline
\end{tabular}

Note: the response scale ranges from 1 (never) to 5 (always) 
Table 3. Correlation between face photoprotection with other indicators of photoprotection

\begin{tabular}{lccc}
\hline & $\mathrm{N}$ & \multicolumn{2}{c}{ Face photoprotection } \\
& & Cloudy & Sunny \\
\hline Frequency of photoprotection whilst outside (1-5) & 66 & 0.72 & 0.61 \\
Avoidance of going outside (1-5) & 66 & 0.01 & 0.06 \\
Body photoprotection & 66 & 0.46 & 0.40 \\
Clinical Rating of adherence (1-20) & 47 & 0.76 & 0.77 \\
Mean daily face photoprotection (0-100\%) & 36 & 0.66 & 0.51 \\
Mean daily facial UVR exposure (SED) & 36 & -0.14 & -0.25 \\
\hline
\end{tabular}


Figure 1. Face regions scored

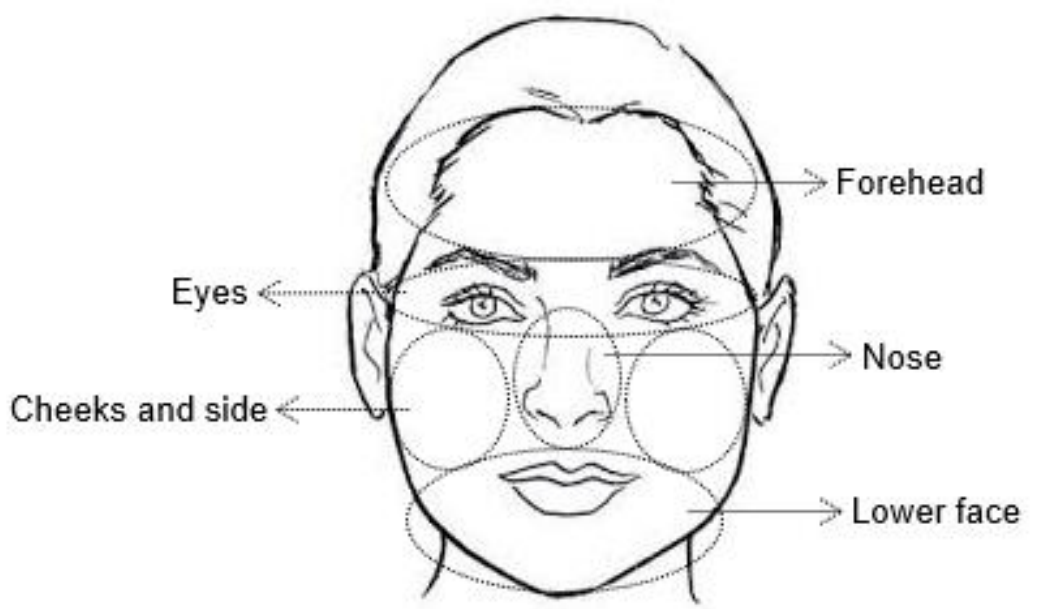


Figure 2. Distribution of categorised face photoprotection scores on cloudy and sunny days

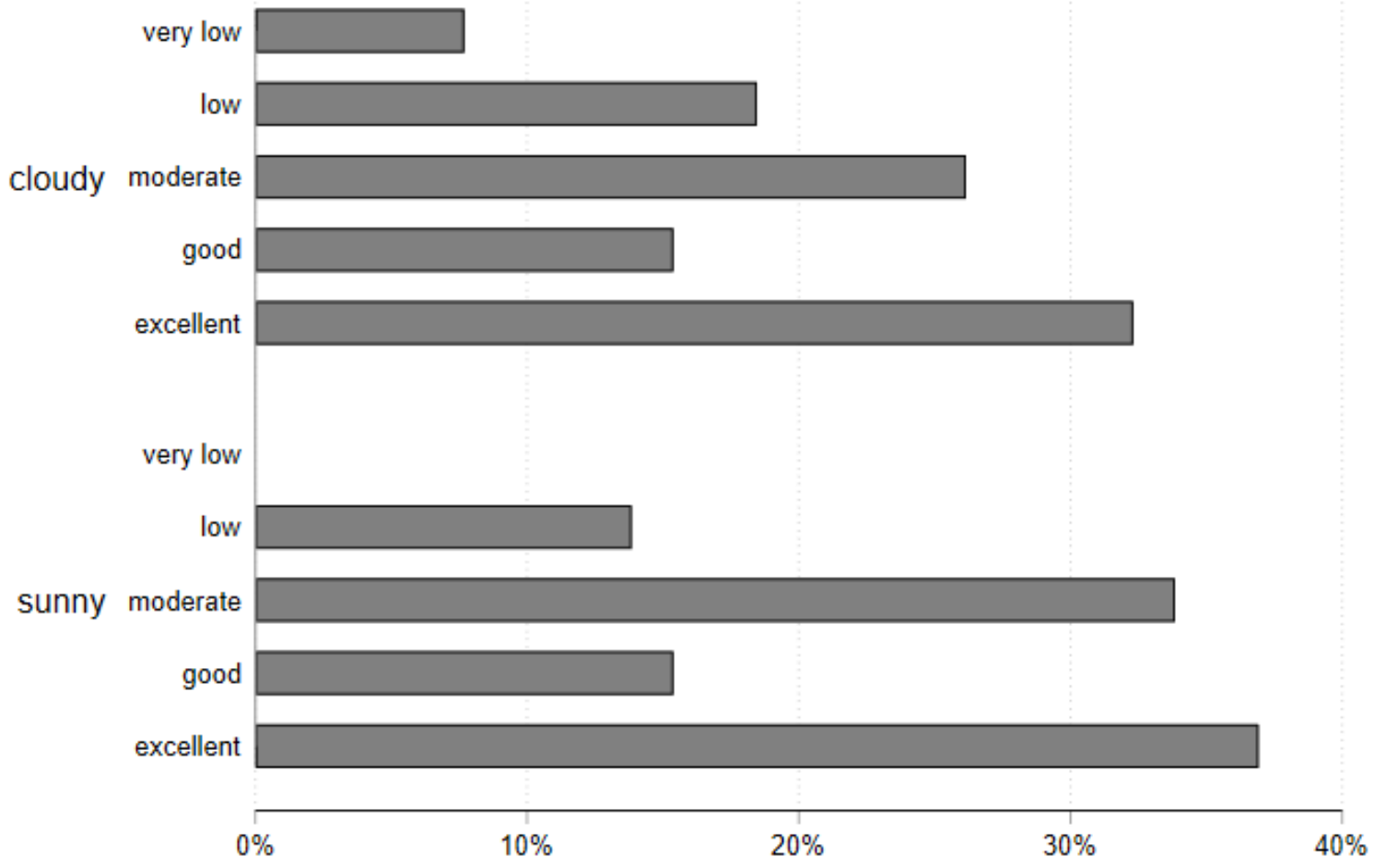


Figure 3. Face photoprotection on cloudy and sunny days for self- and carer-reports

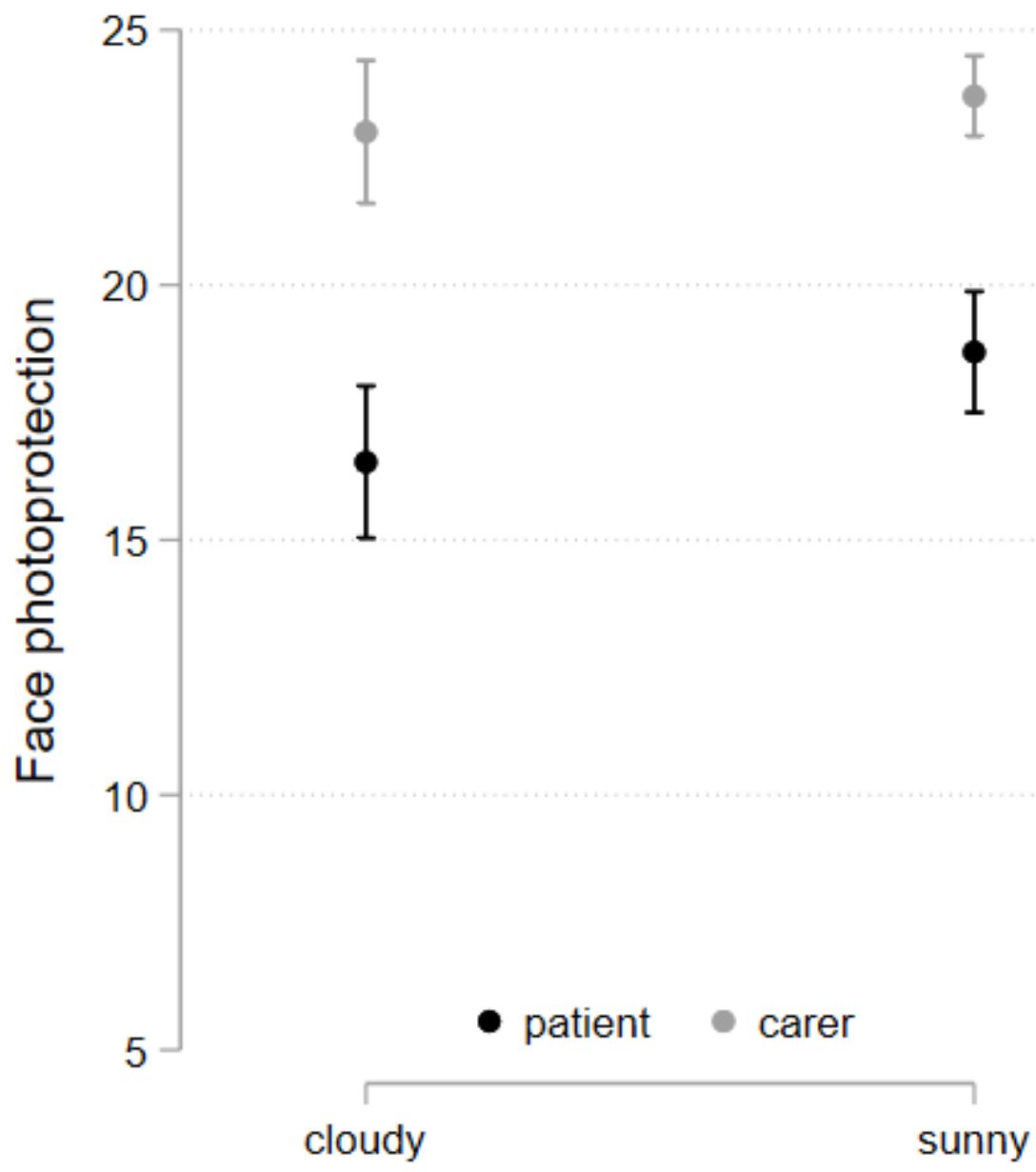


Figure 4. Mean daily face photoprotection and facial UVR exposure by self-reported face photoprotection category
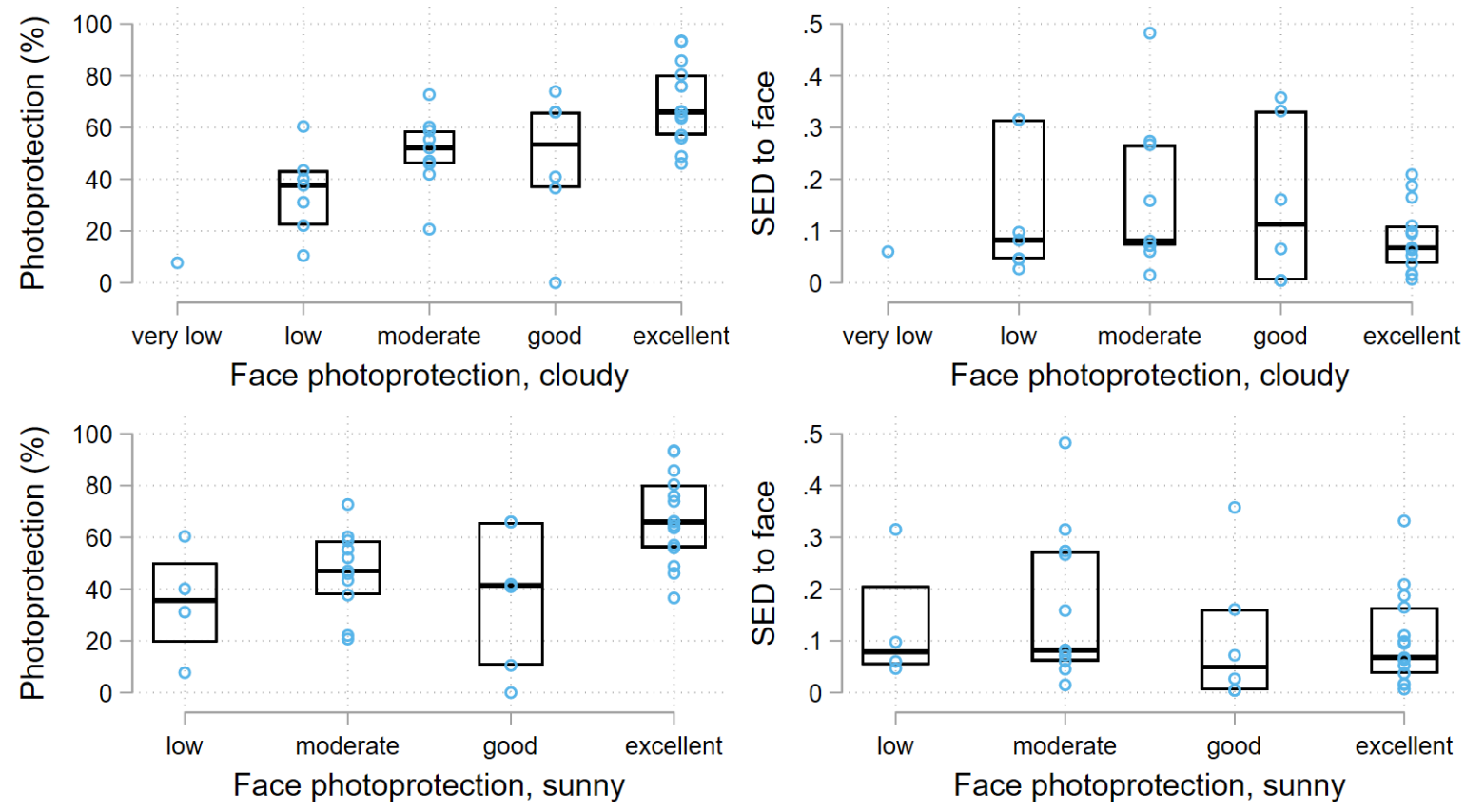